\title{
Operational Concept for Flight Crews to Participate in Merging and Spacing of Aircraft
}

\author{
Brian T. Baxley, ${ }^{*}$ Dr. Bryan E. Barmore ${ }^{\dagger}$ \\ NASA Langley Research Center, Hampton, VA, 23681 \\ Terence S. Abbott ${ }^{\ddagger}$ \\ Booz Allen Hamilton, McLean, VA 22102 \\ William R. Capron \\ Lockheed Martin Corporation, Hampton, VA 22681
}

\begin{abstract}
The predicted tripling of air traffic within the next 15 years is expected to cause significant aircraft delays and create a major financial burden for the airline industry unless the capacity of the National Airspace System can be increased. One approach to improve throughput and reduce delay is to develop new ground tools, airborne tools, and procedures to reduce the variance of aircraft delivery to the airport, thereby providing an increase in runway throughput capacity and a reduction in arrival aircraft delay. The first phase of the Merging and Spacing Concept employs a ground based tool used by Air Traffic Control that creates an arrival time to the runway threshold based on the aircraft's current position and speed, then makes minor adjustments to that schedule to accommodate runway throughput constraints such as weather and wake vortex separation criteria. The Merging and Spacing Concept also employs arrival routing that begins at an en route metering fix at altitude and continues to the runway threshold with defined lateral, vertical, and velocity criteria. This allows the desired spacing interval between aircraft at the runway to be translated back in time and space to the metering fix. The tool then calculates a specific speed for each aircraft to fly while enroute to the metering fix based on the adjusted land timing for that aircraft. This speed is data-linked to the crew who fly this speed, causing the aircraft to arrive at the metering fix with the assigned spacing interval behind the previous aircraft in the landing sequence. The second phase of the Merging and Spacing Concept increases the timing precision of the aircraft delivery to the runway threshold by having flight crews using an airborne system make minor speed changes during enroute, descent, and arrival phases of flight. These speed changes are based on broadcast aircraft state data to determine the difference between the actual and assigned time interval between the aircraft pair. The airborne software then calculates a speed adjustment to null that difference over the remaining flight trajectory. Follow-on phases still under development will expand the concept to all types of aircraft, arriving from any direction, merging at different fixes and altitudes, and to any airport. This paper describes the implementation phases of the Merging and Spacing Concept, and provides high-level results of research conducted to date.
\end{abstract}

\section{Introduction}

The Federal Aviation Administration's current aviation forecast predicts that from 2004 to 2017 the number of passengers will increase $64 \%$, the commercial jet aircraft inventory will increase $73 \%$, and the number of aircraft operations at towered airfields will increase $79 \% .{ }^{1}$ Although commercial jet aircraft comprise only $3.5 \%$ of the total number of active aircraft flying in the National Airspace System (NAS), they will still carry the overwhelming

\footnotetext{
* Research Engineer, Crew Systems and Aviation Operations Branch, MS 152, AIAA Member

${ }^{\dagger}$ Merging and Spacing Principle Investigator, Crew Systems and Aviation Operations Branch, MS 156, AIAA Member

${ }^{\ddagger}$ Senior Research Engineer, Booz Allen Hamilton

$\S$ Senior Research Engineer, Lockheed Martin Corporation
} 
majority of the estimated 1.1 billion passengers in 2017. Much of this increase will undoubtedly have to be absorbed by airports that are not operating close to maximum throughput since most of the major hubs in the United States are already near peak capacity. To consistently and safely achieve near maximum flight operation throughput at these major hubs would provide a measurable benefit. Various factors affect this throughput including airspace design, the spacing interval between aircraft crossing the runway threshold, aircraft ground dwell (taxi time from runway to gate), and the ability of the airport to provide aviation services to the airline operator and passengers.

\section{ATM Concepts to Increase Runway Throughput and Reduce Workload}

An airport operating at $90 \%$ of a nominal maximum rate of 40 Instrument Flight Rules (IFR) arrivals per hour receives 36 aircraft every 60 minutes. A 5\% increase in the arrival rate to maximum service rate ratio allows two more aircraft per hour to land and, furthermore, queuing theory states that increase will generate a system wide $50 \%$ decrease in delay for aircraft in the arrival stream. ${ }^{2}$ Current procedures generally have aircraft descend to a lower altitude where the speeds of the various aircraft are similar, and then power, drag, or vectoring is used to create the desired arrival sequence and spacing between aircraft. This procedure incurs an additional penalty in fuel, time, and noise for the aircraft operator and, generally, also a reduction in runway throughput.

Several avenues are available to increase the aircraft arrival rate and decrease delay. These include:

1) use suitable but under-utilized airfields

2) build new runways (increase in airport throughput, but expensive and environmental challenges, and overall throughput may be hindered by airspace or ground movement constraints)

3) reduce wake vortex separation criteria (use research data to reduce the landing interval, increasing the maximum IFR rate)

4) reduce the separation buffer added to the minimum separation criteria between aircraft (flight operations would be closer to the established maximum IFR rate).

Extensive simulator and flight trial research over the past 30 years by National Aeronautics and Space Administration (NASA) ${ }^{3,4,5,6,7,8,9}$, MITRE ${ }^{10,11}$, and Eurocontrol ${ }^{12,13}$ has been conducted to improve runway arrival rates via en route metering and self-spacing of aircraft. A central element of much of this research is an advanced ground tool for the Air Traffic Controller (ATC) that determines an appropriate arrival schedule and landing time interval between aircraft, then computes the appropriate speed required to space aircraft close to the minimum time or distance allowed for the runway conditions. A separation buffer is added to this interval to reduce the likelihood that IFR separation criteria will be compromised or that an aircraft will have to execute a missed approach because of insufficient spacing. This separation buffer is added to compensate for differences in aerodynamic characteristics of various aircraft as they slow down and configure, and differences in the pilot's or controller's operational technique. Although the required minimum spacing interval and the additional buffer data are not currently collected by the FAA, past research indicates the buffer, or that time above the minimum required for safe separation during IFR operations, is approximately 20 to 30 seconds. ${ }^{14}$ Simulations of the Merging and Spacing Concept at NASA Langley indicate flight crews can safely and routinely delivers their aircraft to the runway threshold within 5 to 10 seconds of the assigned time given the appropriate information and procedures. ${ }^{15}$ This precise delivery of aircraft yields a 10 to 15 second reduction in the separation buffer and equates to at least a $10 \%$ increase in throughput for a nominal 120 second IFR separation criteria (5 nautical mile separation at 150 KIAS final approach speed approximates 120 seconds).

In addition to increasing runway throughput, the Merging and Spacing Concept has tremendous potential to reduce fuel consumption and noise. Furthermore, the accuracy and precision of the Merging and Spacing Concept will enable tailored Continuous Descent Arrivals for further fuel savings and noise reductions.

\section{Merging and Spacing Concept}

The Merging and Spacing Concept development is being led by the Federal Aviation Administration (FAA), with significant membership and participation from MITRE, UPS, NASA, Aviation Communication and Surveillance Systems (ACSS), and Eurocontrol. This concept is designed to merge arriving aircraft from different altitudes and directions during the en route cruise and then deliver that arrival stream precisely to the runway threshold using optimized flight profiles with minimal speed changes. The ability of each aircraft to fly closer to its optimum flight profile is of vital interest to airline operators since Continuous Descent Arrivals (CDAs) can now be flown with minor speed adjustments to precisely deliver aircraft at the runway threshold, realizing fuel savings per aircraft while maintaining system efficiency. The Merging and Spacing Concept uses elements of previous research efforts and builds on them by combining or refining the following: 
- the use of four dimensional (4D) trajectories for the accurate calculation of the runway Estimated Time of Arrival (ETA) to generate the arrival sequence, and to enable the merging of multiple arrival streams from any direction,

- the establishment of an optimum arrival stream sequence and the desired spacing interval between aircraft during the en route phase of flight,

- the dissemination of the arrival sequence (in this concept, the aircraft to follow) and spacing to the flight crew who actively participate in implementing the plan,

- the aircraft's optimum trajectory and airspeed required to achieve the assigned spacing at the runway threshold is calculated onboard the aircraft for very precise aircraft delivery,

- transitions from a scheduled Required Time of Arrival (RTA) to a relative spacing behind the lead aircraft, providing more flexibility for system error and a possible reduction in the separation,

- the compensation for differences in final approach speeds of various aircraft types by calculating an appropriate time offset for the trailing aircraft to create maximum throughput at the runway threshold, and

- the use of broadcast aircraft state data to correct forecasted winds errors to improve runway ETA calculations.

The combination of these characteristics in the Merging and Spacing Concept creates a predictable, efficient, and precise stream of aircraft, which can enable a reduction in the separation buffer between aircraft, allowing a 5 to $10 \%$ increase in runway throughput. ${ }^{16}$ The following sections describe the current and potential phases of the implementation plan. A summary is provided in Table 1, with the significant changes highlighted by bold text.

Table 1. Summary of Phased Implementation Plan for Merging and Spacing Operations

\begin{tabular}{|l|l|l|l|l|}
\hline & \multicolumn{1}{|c|}{ Phase I } & \multicolumn{1}{c|}{ Phase II } & Proposed Phase III & Proposed Phase III+ \\
\hline Air Carrier & UPS & UPS & $\begin{array}{l}\text { Ans equipped } \\
\text { aircraft / airline }\end{array}$ \\
\hline Airport & Louisville, KY & Louisville, KY & Louisville, KY & Multiple airports \\
\hline $\begin{array}{l}\text { Sequence and } \\
\text { interval spacing } \\
\text { tool }\end{array}$ & $\begin{array}{l}\text { Located in and } \\
\text { operated by Airline } \\
\text { Operations Center }\end{array}$ & $\begin{array}{l}\text { Located in and } \\
\text { operated by Airline } \\
\text { Operations Center }\end{array}$ & $\begin{array}{l}\text { Located in and } \\
\text { operated by Airline } \\
\text { Operations Center }\end{array}$ & $\begin{array}{l}\text { Located in and } \\
\text { operated by Air } \\
\text { Traffic Control }\end{array}$ \\
\hline $\begin{array}{l}\text { Direction of } \\
\text { arrival }\end{array}$ & $\begin{array}{l}\text { Limited to } \\
\text { westbound arrivals }\end{array}$ & $\begin{array}{l}\text { Limited to } \\
\text { westbound arrivals }\end{array}$ & Multiple directions & Multiple directions \\
\hline Merge points & Single, at altitude & Single, at altitude & $\begin{array}{l}\text { Multiple, at altitude } \\
\text { \& in TRACON }\end{array}$ & $\begin{array}{l}\text { Multiple, at altitude } \\
\text { \& in TRACON }\end{array}$ \\
\hline $\begin{array}{l}\text { Arrival sequence } \\
\text { and spacing } \\
\text { interval }\end{array}$ & $\begin{array}{l}\text { Calculated by } \\
\text { ground tool, data- } \\
\text { linked to flight crew }\end{array}$ & $\begin{array}{l}\text { Calculated by } \\
\text { ground tool, data- } \\
\text { linked to flight crew }\end{array}$ & $\begin{array}{l}\text { Calculated by ground } \\
\text { tool, data-linked to } \\
\text { flight crew }\end{array}$ & $\begin{array}{l}\text { Calculated by } \\
\text { ground tool, data- } \\
\text { linked to flight crew }\end{array}$ \\
\hline Speed Commands & $\begin{array}{l}\text { Calculated by } \\
\text { ground tool, data- } \\
\text { linked to flight crew }\end{array}$ & $\begin{array}{l}\text { Calculated initially } \\
\text { by ground tool, then } \\
\text { onboard aircraft }\end{array}$ & $\begin{array}{l}\text { Calculated initially by } \\
\text { ground tool, then } \\
\text { onboard aircraft }\end{array}$ & $\begin{array}{l}\text { Calculated initially } \\
\text { by ground tool, then } \\
\text { onboard aircraft }\end{array}$ \\
\hline
\end{tabular}

\section{Phase I Implementation}

Phase I implementation allowed the Airline Operations Center (AOC) to calculate and datalink a speed to the flight crew for them to fly so as to arrive properly spaced in the right sequence at an en route metering fix. The objectives were to assess the feasibility and usability of the Merging and Spacing operations for both controllers and flight crew without requiring new software or procedural changes for either ATC or the flight crews. Successful implementation of this phase of the Merging and Spacing operation would demonstrate a predictable arrival stream that required little intervention by ATC, produced an increase in runway throughput (less variance from desired spacing interval), and reduced fuel consumption (no low-altitude vectoring).

Flight trials were conducted in 2006 at Louisville, KY, by the United Parcel Service's AOC in conjunction with the FAA, the Louisville TRACON, Kansas City Air Route Traffic Control Center (ARTCC), and Denver ARTCC. Ten to twelve east-bound UPS aircraft to Louisville participated over several nights, all equipped with the ability to transmit and receive information from the AOC. Once these aircraft were established at cruise altitude, the AOC ground tool used datalinked aircraft data to establish a sequence to a metering fix. The datalinked instructions allowed the flight crew to fly close to their optimum speed, and then determined a time interval between aircraft that 


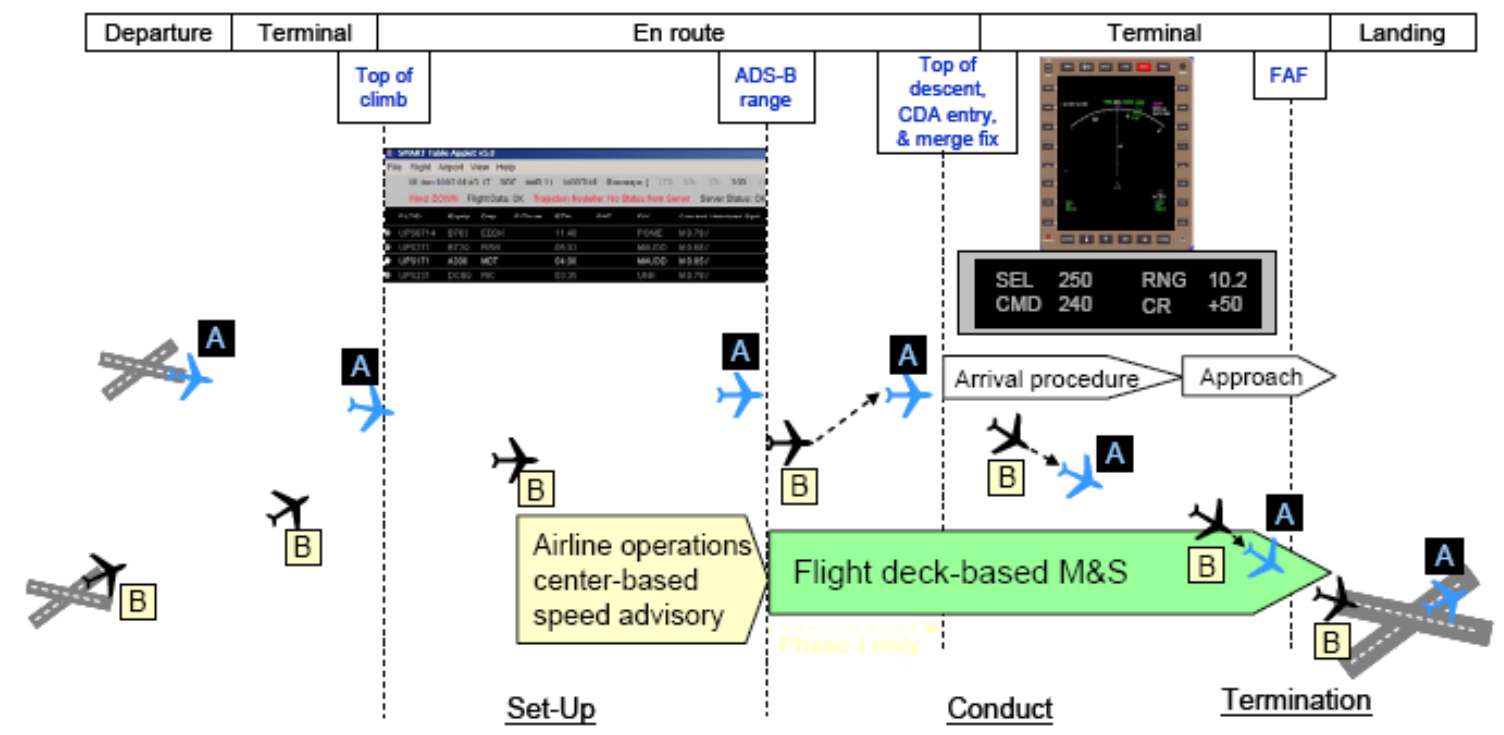

Figure 1. Phases of the Merging and Spacing Concept

met wake vortex and miles-in-trail requirements from ATC (see yellow arrow in Figure 1). A speed was then calculated by the AOC ground tool for each aircraft in the arrival stream to reach the metering fix in the correct sequence with the appropriate time interval behind the lead aircraft. This speed and any required updates were then datalinked to the flight crew (the change in speed was generally very small and any ATC instruction took precedence). Flying the assigned speed to the single metering fix allowed the ten to twelve aircraft to merge from various directions in the desired sequence and with the assigned spacing interval.

An FAA approved special arrival procedure was created for the flight trial, which consisted of a Standard Terminal Arrival Route (STAR) from the en route metering fix to the runway threshold, with steeper descent angles to approximate a CDA and specified speeds for each segment. Flying a published arrival route and speed allowed the flight crew to maintain the interval spacing between the aircraft from the metering fix to the runway. No new software or equipment was required for ATC or flight crews, and all IFR procedures were followed. Although research is still necessary to determine where the optimum point to begin calculating and issuing speed commands for the aircraft is, the flight test explored ranges of 300 to 1000 nautical miles from the destination airport. Initial results indicate the flight crews participating in the UPS flight trial were able to fly the ground tool assigned speed to achieve the desired sequence and spacing between aircraft by the metering fix, and maintain it through landing on the runway. Feedback from the AOC, flight crew, and controllers was very positive. Results will be published in 2007 by MITRE and NASA Ames.

\section{Algorithm Logic for Ground-Based Spacing in Phase I}

Several tools were used in Phase I by the AOC at various ranges from the destination runway. NASA Ames developed the Cruise Speed Calculator (CRZ), a simple and easy to implement tool that estimated a time of arrival based on the aircraft's broadcast position and speed. CRZ estimated the time of arrival by assuming the aircraft proceeded from its current position direct to the en route metering fix that served as the merge point and did not use flight plan data or en route wind information in the calculations. The software created a logical arrival sequence and inserted an appropriate time interval between each aircraft (based on wake vortex criteria and runway acceptance capacity), then calculated a speed to meet the arrival schedule. Changes of the aircraft's initial speed were generally small, and required less than a 0.02 Mach change for aircraft 300 to 600 nautical miles from Louisville.

During the UPS flight test in May 2006, the Self Managed Arrival Resequencing Tool (SMART) from MITRE was run concurrently with CRZ to explore the impact of a more sophisticated and complex tool. The SMART software calculated a sequence to merge point or metering fix but incorporated flight plan and wind data to provide a more accurate estimation. It also ensured an appropriate time interval between each aircraft, calculated the speed required to arrive at the fix at that time, and then uplinked that speed to the aircraft for the flight crew to execute. 
Figure 2 shows the display generated by the prototype SMART software used by the UPS AOC during the May 2006 flights to calculate the desired speed for each participating aircraft. Results from the May 2006 flight and the October 2006 field test flights are planned to be published by MITRE and NASA Ames in 2007

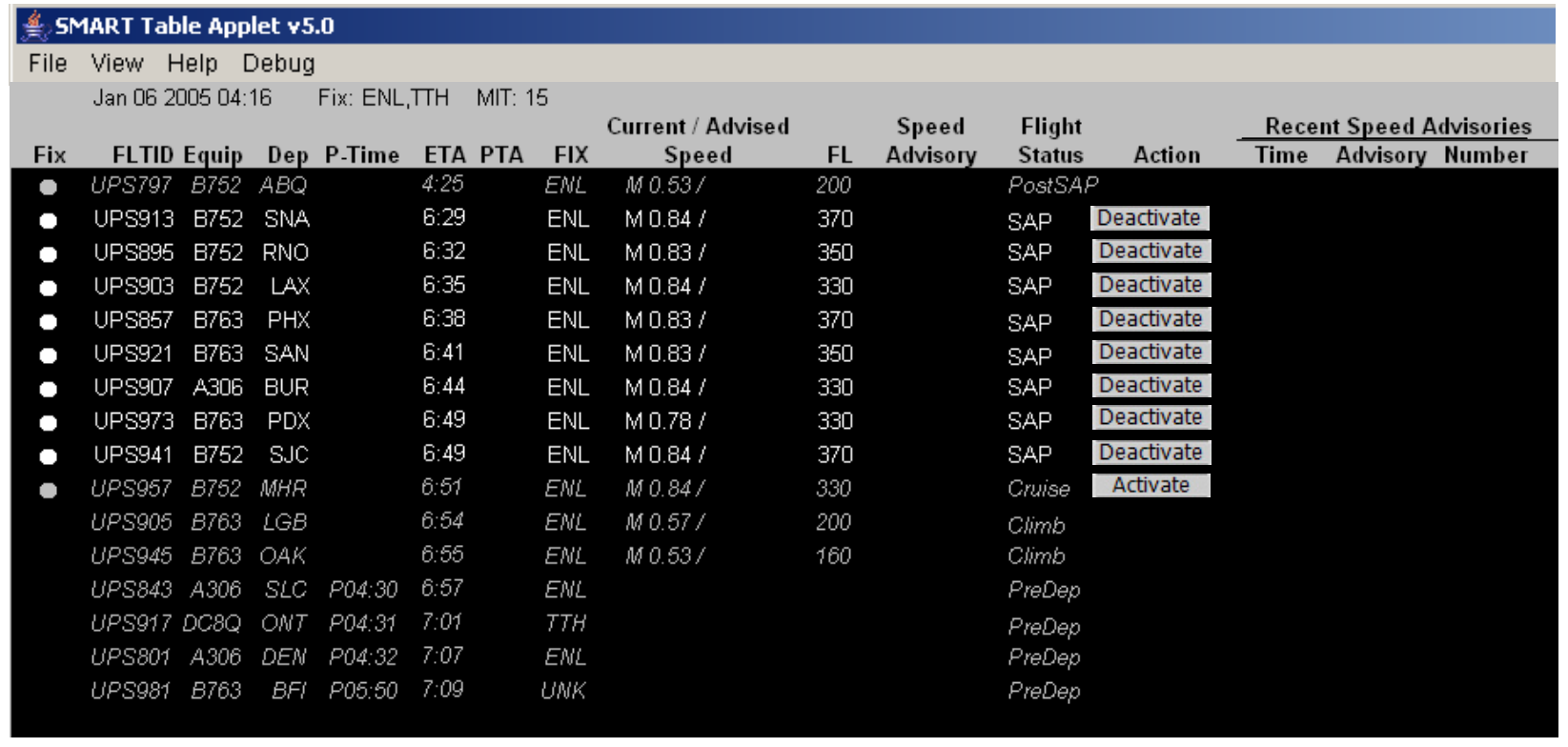

Figure 2. Prototype Self Managed Arrival Resequencing Tool (SMART) used in UPS flight trial.

\section{Phase II Implementation}

Phase II builds on Phase I by using the ground tools and procedures developed in that phase, then adding a capability onboard the aircraft to allow flight crews to fly precise speeds to achieve the assigned spacing interval between aircraft. The objective of the Phase II implementation is to improve the precision of aircraft delivery to the runway threshold over Phase I by 5 to 10 seconds per aircraft. This would allow for a reduction in the separation buffer added to the minimum spacing interval between aircraft, thereby increasing runway throughput. This increase in precision is accomplished by having flight crews select the preceding aircraft in the sequence, or Traffic To Follow (TTF), and then make minor speed changes computed by onboard software that uses broadcast aircraft state data to achieve and maintain the assigned spacing interval (shown by the green arrow in Figure 1).

In Phase II, the initial portion of the flight remains unchanged from the Phase I description. A centralized software tool operated by the AOC receives aircraft data and route information from all aircraft proceeding to the runway of interest. Sometime after the aircraft reaches cruise altitude, the ground tool calculates an estimated time of arrival to an en route metering fix. The ground tool creates an arrival sequence based on broadcast aircraft state data and its $4 \mathrm{D}$ trajectory flight plan, and also determines the required spacing between aircraft based on runway surface conditions, wake vortex category, and ATC miles-in-trail requirements. The tool then calculates the speed each aircraft needs to fly to arrive at the metering fix in the desired sequence with the appropriate spacing interval.

The added feature of the Phase II implementation is the onboard calculation of the appropriate speed to achieve the assigned spacing. Research is ongoing, with several key questions to be addressed, including:

1) Does the aircraft arrival sequence need to be fixed (or frozen) by the ground tool prior to commencing flight-deck based Merging and Spacing? If so, the appropriate location to begin using the airborne software may be closer to the airport, perhaps around 200 to 300 nautical miles from the runway. If not, in some situations it may be beneficial to commence flight-deck based spacing considerably farther out.

2) What effect does the ground tool's conditioning of the arrival stream have in the Merging and Spacing Concept? If the flight crew arrives at the metering fix within 30 seconds of the assigned time and then flies the published arrival route and speeds, can the assigned time interval between aircraft provide separation from the metering fix to runway threshold? Conversely, what is the impact and benefit or cost if there is no ground tool to calculate a speed to precondition the arrival stream, and spacing is accomplished through the flight-deck based software by flight crews implementing directions from the AOC or ATC? 
3) What information and what terminology are required for the flight crew to positively identify the assigned Traffic To Follow? What information and terminology are required for the AOC or ATC to verify that the flight crew has selected the correct Traffic To Follow?

4) What are the acceptable operational limits in the magnitude of change in speed, and the absolute speeds calculated by the ground tool and by the airborne tool? How closely do the two tools need to align with each other for a given set of parameters?

In upcoming flight trials planned for Phase II, the intent is to have participating UPS aircraft fly the commanded ground tool speed to arrive at the metering fix within one minute of their assigned time. As in the Phase I flight trials, this should merge the aircraft from various arrival streams at that fix. At some point prior to the merge at the metering fix, information regarding the Traffic To Follow and the spacing interval behind the TTF is data-linked to the flight crew. Once the TTF surveillance data is available on the aircraft, the flight-deck based software can calculate the required speed to be flown to achieve the assigned time interval behind the TTF. Past research indicates having the speed calculated on board the aircraft produces quicker and more accurate speed commands, and therefore allows flight crews to fly a speed that more precisely deliverers the aircraft to the runway at the desired time. ${ }^{7,15-17}$

Initial feedback from pilots during simulation studies indicate no significant change in workload is required to achieve the significant improvement in precision of aircraft delivery to the runway. Results from experiments conducted by MITRE and NASA indicate flight crews can consistently deliver aircraft to the runway within 5 seconds of the desired time, creating the potential to reduce the separation buffer added to minimum separation criteria, thereby increasing runway throughput.

\section{Algorithm Logic for Flight-Deck Based Spacing in Phase II}

Several key decisions were made in the development of the speed guidance imbedded in the flight-deck based software. The Airborne Spacing for Terminal Arrival Routes (ASTAR) algorithm created at NASA Langley is capable of spacing aircraft based on an absolute schedule or on a relative interval behind the preceding aircraft, and that interval can be assigned as time or as distance. To maintain a predictable and stable arrival flow and minimize workload increases for the flight crew, the onboard spacing software is limited to making small speed corrections, with the nominal command speed being that of the speed schedule for that segment of the Standard Terminal Arrival Route (STAR). Filtering and gain scheduling techniques are also used to reduce or inhibit speed corrections based on the distance the aircraft is from the runway (Figure 3).

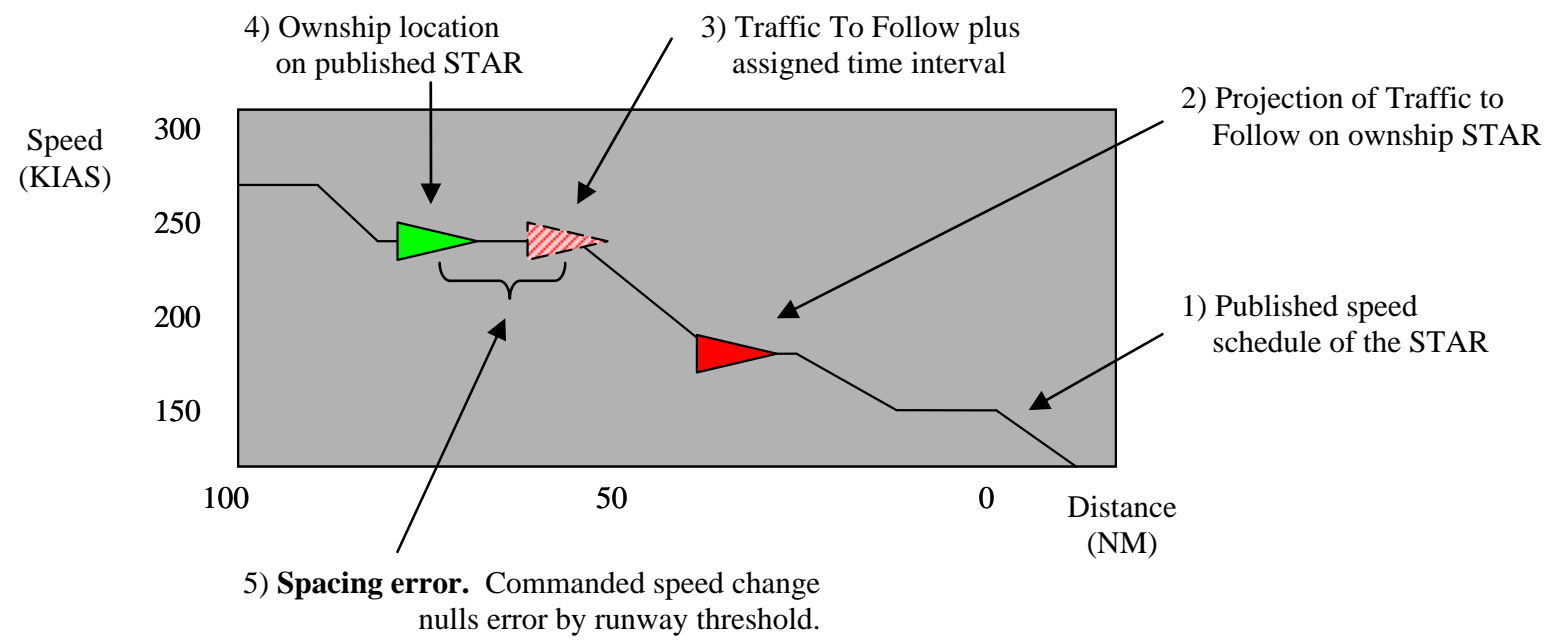

Figure 3. Speed command calculation using aircraft location, a known trajectory and speed.

Furthermore, other than wind prediction error, ASTAR can compensate for one of the greatest influences in variance from desired arrival time, that being the different final approach speeds flown by various types aircraft at diffeering weights during the final portion (typically the last 5 miles) of the instrument procedure. The ASTAR software uses the lead aircraft's final approach speed, obtained either via datalink from the ground or broadcast as part of the leading aircraft's surveillance message, and its own final approach speed to calculate a time offset at the 
Final Approach Fix (FAF). This offset allows the following aircraft to discontinue spacing at the FAF and decelerate to its planned final approach speed while still landing at its assigned time interval.

\section{Beyond Phase II Implementations}

The intent of these proposed follow-on implementation phases is to transition from single to multiple carriers, and to accommodate multiple arrival directions and different types of aircraft. The most immediate step along this path is to expand the UPS flight operations at Louisville to include opposite direction arrivals, and multiple merge points either at en route altitudes or lower in the TRACON. The multiple arrival routes and directions will allow different types of aircraft to fly their optimum en route speeds and altitudes, as well as fly closer to their optimum CDA. Accommodating aircraft with different aerodynamic characteristics can be accomplished by having aircraft with slower cruise or descent speeds merge at a later point on the approach, perhaps as late as the FAF. This phase of the Merging and Spacing Concept should significantly increase the number and type of aircraft that will immediately benefit from savings in fuel consumption and time.

The desired end goal is to deploy the Merging and Spacing operation to any airline at any airport that would benefit from these operations. Key developmental challenges will include migrating the ground tool from the AOC to ATC to accommodate multiple airline operations, and producing airborne software and equipment at an affordable price.

\section{Experiment Results for Flight Crew Merging and Spacing Aircraft}

A fast-time batch study at NASA Langley was completed in February of 2006 as an initial, exploratory evaluation of the concept in Phase III and beyond. The study examined the behavior of 40 aircraft using the ASTAR algorithm in four arrival streams to three different merge points, flying a CDA to Runway 18R at Dallas Forth Worth airport (Figure 4). Three aircraft types at various enroute altitudes and speeds began the scenario 350NM from the runway according to a randomized schedule. To test the algorithm, the 40 aircraft were initialized with some amount of error relative to the scheduled arrival time, which the algorithm had to resolve by runway threshold. Three key variables selected from a much larger list were studied to determine the impact on the arrival stream.

These variables were the effects of error in the wind forecast (up to 15 knots between actual and forecast), the effect of a delay by the flight crew in changing speeds according to the ASTAR speed command (30 seconds above 11,000' MSL and 8 seconds below), and the impact a disruption had on the entire aircraft arrival flow (the assigned time interval for the fifth aircraft behind the fourth was increased by one minute several minutes into the scenario).

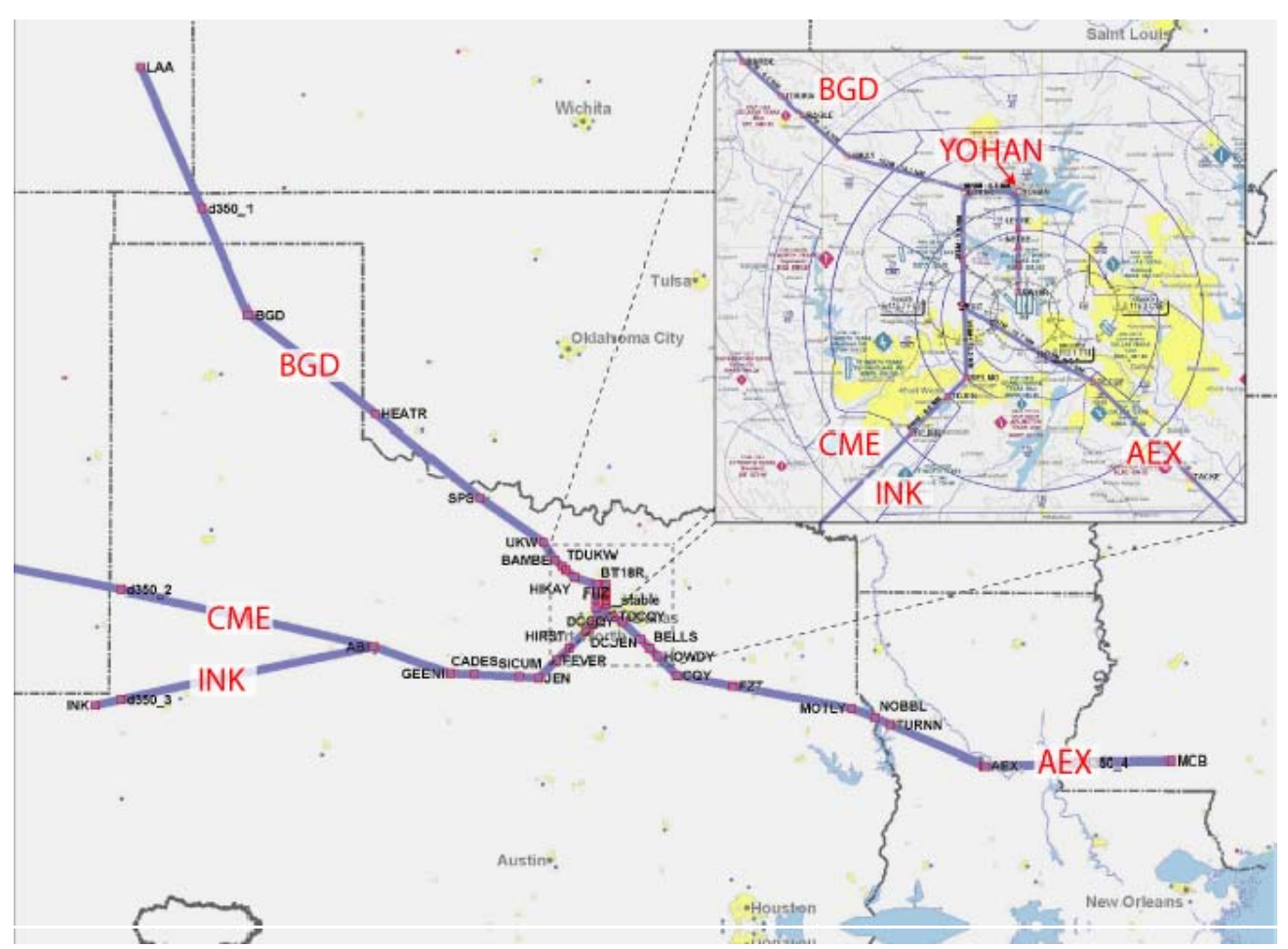

Figure 4. Arrival routes and Constant Descent Approaches to Runway 18R at Dallas Fort-Worth. 
Selected highlights are presented in this section with complete information planned to be published next year. Initial results of this experiment show high correlation with past Langley experiments. ${ }^{17,18,19}$ Figure 5 shows the behavior of the $26^{\text {th }}$ and $27^{\text {th }}$ aircraft in the middle of a 40 aircraft arrival stream merging in a nominal scenario. The momentary increase in airspeed at Top of Descent at the TRACON boundary (FL110) is due to the difference between ASTAR algorithm calculations and the aircraft's Flight Management System calculations.
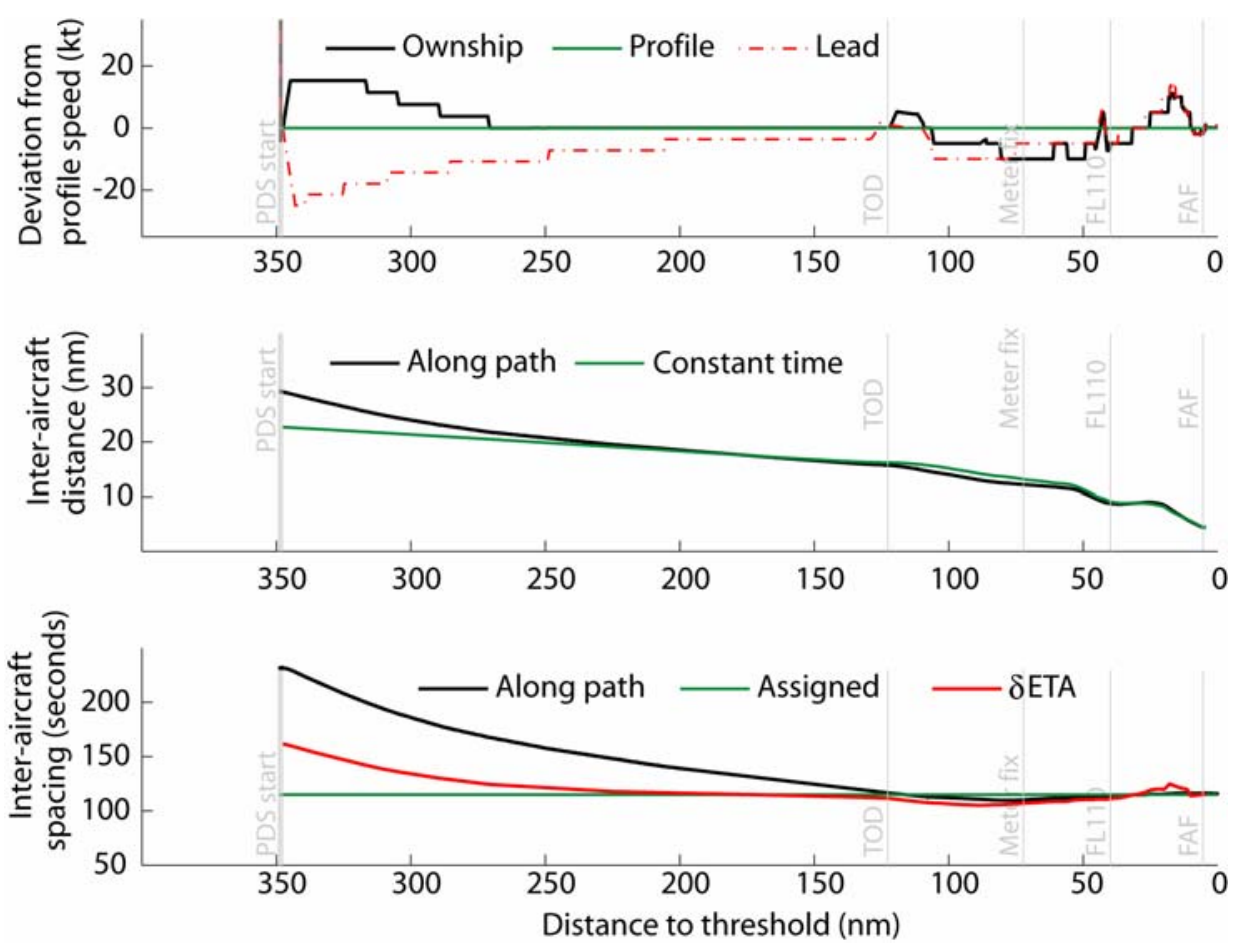

Figure 5. Speed and spacing of aircraft in arrival stream using flight-deck generated speed commands.

Figure 6 is a summary of the spacing between each of the 40 aircraft in one of the scenarios, demonstrating how the arrival stream slowly moves to the assigned spacing interval between aircraft at the runway threshold. This graph shows spacing deviation as a difference between aircraft's current estimated time of arrival and desired time of arrival (assigned time interval behind TTF at published profile speed) at the runway threshold. The spacing tool gradually causes the aircraft to converge on the assigned interval instead of aggressively achieving it early to reduce flight crew workload and increase the stability of the entire Merging and Spacing operation.

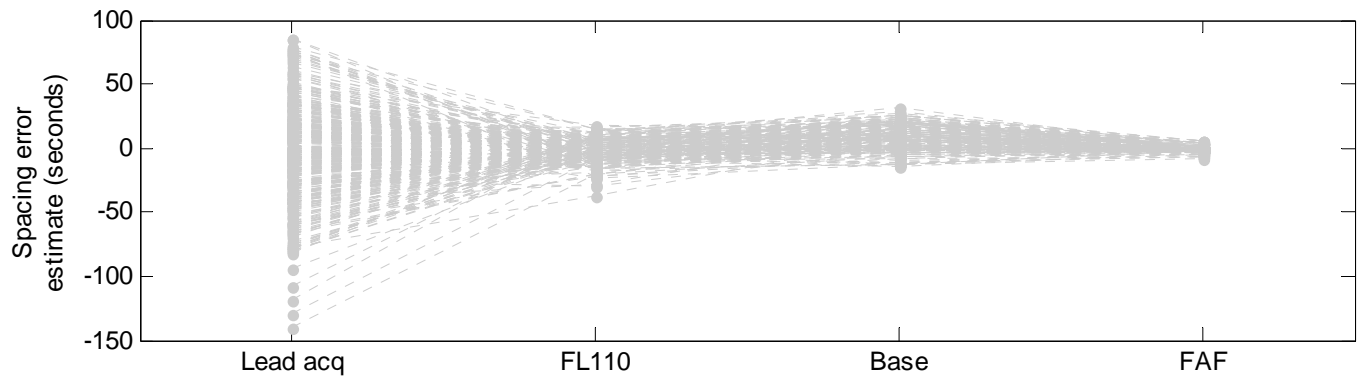

Figure 6. Gradual reduction in deviation from assigned spacing interval over remaining trajectory.

Figure 7 shows the Merging and Spacing operation's potential to precisely delivery aircraft to the runway at the desired interval, even given large spacing deviations at initialization. The open circles show the estimated spacing 
interval error when the spacing tool was first initialized (about 10 seconds after entering the simulation). The spacing interval error is the difference between the ownship's initial ETA and the lead's own ETA at the same time. Since aircraft approaching from different directions are initially outside surveillance (ADS-B) range, they follow the reference trajectory until coming within ADS-B range $(120 \mathrm{~nm})$ of their lead. The estimated error in spacing when their lead is acquired is shown as the solid circles. The change between the initial value and when the lead is acquired is largely a result of different winds experienced along different routes. The open square shows the final spacing deviation at the threshold. Even large differences of up to 100 seconds (350 nautical miles from the runway) could be overcome by the runway threshold giving final differences of only a few seconds.

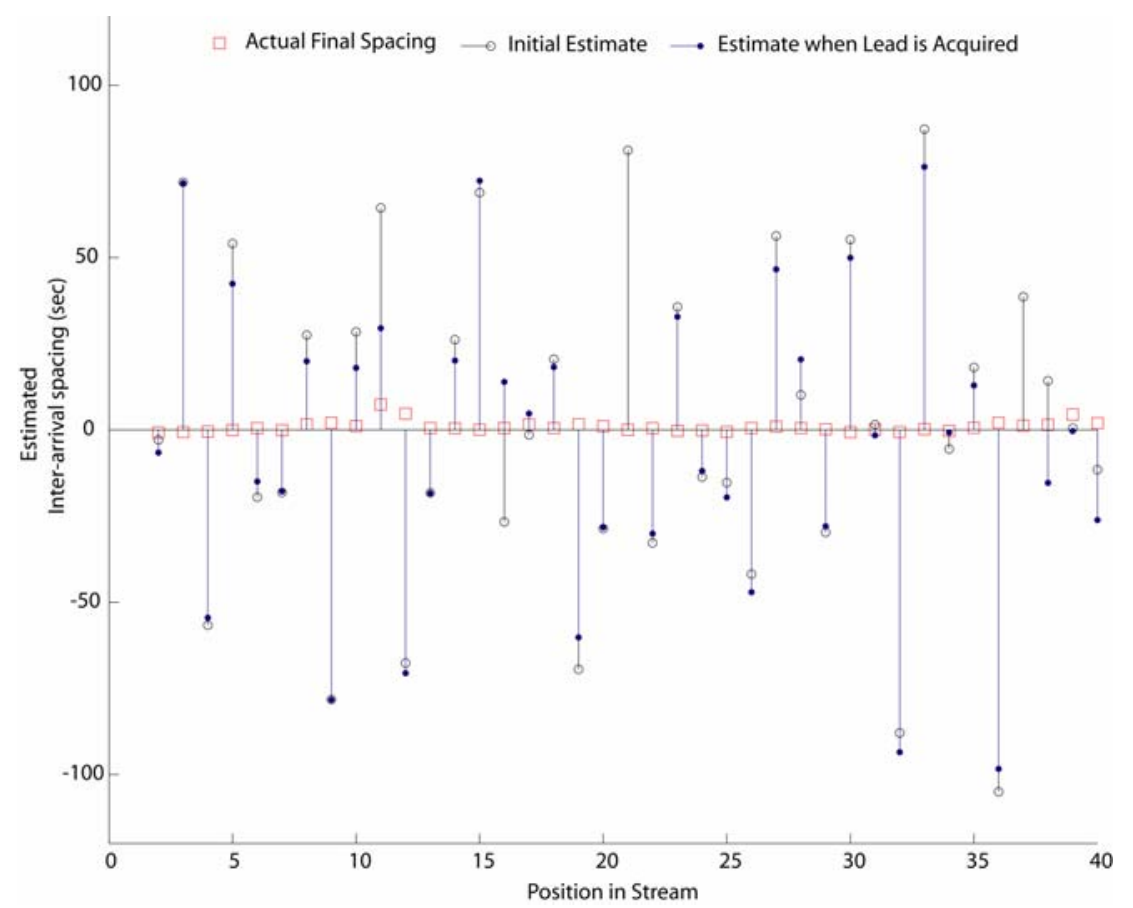

Figure 7. Spacing interval error between aircraft at initialization and at runway.

Key design requirements of the flight-deck based spacing algorithm that appear to have been satisfied in this first cursory experiment include:

- Predictable behavior very similar to today's flight procedures from both a flight crew and ATC point of view, i.e., the speed changes required by the flight crew are small and generally 5 knots or less.

- Little change in flight crew workload, inferred from the batch study results of an average of one speed change per six minutes during the en route phase of flight, and once every minute during descent

- $\quad$ Meets current IFR requirements, i.e., there were no aircraft separation violations.

\section{Issues Requiring Further Research}

\section{A. Expected non-normal procedures}

The following operational areas require further research in the Merging and Spacing Concept and are planned for the future:

1) Loss of CDTI or incoming aircraft surveillance data

2) Loss of outgoing aircraft surveillance data

3) Runway or airfield configuration change

4) Assigned spacing interval generates speed command above/below desired or aircraft limit

5) Flight crew selects wrong Traffic To Follow

6) Traffic To Follow is vectored off broadcast 4D trajectory 
7) Ability to maintain minimum separation criteria in question

8) Datalink reliability and bandwidth availability and any impact on procedures

9) What information needs to be displayed to the flight crew to enable compliance with the procedure

\section{B. Mixed equipage operations}

The current Merging and Spacing procedures allow a fully equipped aircraft (ADS-B in and out, datalink, CDTI, trained flight crew) to follow other minimally equipped aircraft (ADS-B out only), or any surveillance target visible on CDTI (assuming that the trajectory of that aircraft is known). Exploratory work with controllers and pilots indicates there may be a possibility of limited participation of aircraft without ADS-B out equipment (results of 2006 study at NASA Ames to be published in 2007). Notwithstanding that possibility, procedures need to be developed to handle the scenario of high density, mixed equipage operations that are expected.

\section{Sensitivity studies}

The airborne self-spacing algorithm could maintain separation between aircraft once established, but should still minimize speed changes to maintain predictability and reduce workload. The possibilities range from a one knot speed changes (auto-throttles using frequent speed command changes) to no speed command given until a fifteen knot speed change (fewer speed changes) is required. Testing of the current ground and airborne algorithms yielded 2 to 5 knot speed change commands every 60 to 70 seconds, however further studies are needed to determine the acceptable rate of speed command changes to the flight crew and the impact on system stability and aircraft separation.

Another study is to optimize the rate at which the algorithm attempts to achieve the desired spacing interval. The algorithm includes the provision to rapidly achieve the desired spacing rather than by the runway threshold. Research is needed to determine if the increased rate of correction increases ATC or crew workload, is acceptable to the flight crews, and if there is a benefit or cost to this approach.

\section{Conclusion}

The Merging and Spacing Concept has the ability to realize at least a 5\% increase in runway throughput, and enable CDAs that yield reductions in fuel consumption and noise. The concept achieves this by precisely delivering an arrival stream to the runway within a very small time buffer of the assigned time interval. To achieve this, the Merging and Spacing Concept employs a ground tool using 4D trajectories for accurate estimation of runway arrival time, establishes an arrival sequence for all arrivals from any direction or altitude, and determines the appropriate time interval between aircraft. The tool then calculates a speed for the aircraft to fly to achieve the desired spacing. That speed, as well as the RTA to the runway, Traffic To Follow, and time interval between the aircraft, are datalinked to the flight crew. The flight crew fly the datalinked airspeed until the Traffic To Follow information is available on their CDTI equipment, then use onboard speed to achieve the desired sequence and interval. This increase in precision delivery allows the separation buffer to be reduced, generating the $5 \%$ increase in throughput, and the onboard calculations of trajectory and speeds enables more optimum profiles to be flown for savings in fuel consumption and reductions of noise.

\section{Acknowledgements}

The authors would like to recognize the entire Merging and Spacing Working group chaired by the FAA and MITRE for their work over the past three years. In particular, John Marksteiner (FAA) and Randy Bone (MITRE) as Co-Chairmen have been pivotal in organizing the research work, and Captains Robert Hilb and James Walton (UPS) for providing their operational expertise. Researchers Vernol Battiste, Nancy Smith (both at NASA Ames) and Peter Moertl (MITRE) were instrumental in their work developing and testing the first phase of the Merging and Spacing Concept, and David Williams (NASA Langley) for providing his expertise in Continuous Descent Arrivals. And many others in the group have made significant contributions in providing their expertise in Air Traffic Control, flight crew procedures, and FAA regulatory requirements. 


\section{References}

1 FAA Aerospace Forecasts, Fiscal Years 2006-2017, U.S. Department of Transportation, FAA, Office of Policy and Plans, http://www.faa.gov/data_statistics/aviation/aerospace_forecasts/2006-2017/ [cited 21 Aug 2006]

2 Credeur, L., "Basic Analysis of Terminal Operation Benefits Resulting from Reduced Vortex Separation Minima”, NASA TM-1977-78624

3 Credeur, L., Capron, W., "A Description and Evaluation of “TIMER” - A Time-Based Terminal Flow-Control Concept”, NASA TP-1991-10996

4 Abbott, T., "Flight Test Evaluation of the Airborne Information for Lateral Spacing (AILS) Concept", NASA TM-2002-211639

5 Callantine, T., Lee, P., Mercer, J., Prevot, T., “Terminal-Area Traffic Management with Airborne Spacing”, AIAA 2005-7403

6 Abbott, T., "Speed Control Law for Precision Terminal Area In-Trail Self Spacing”, NASA TM-2002-211742

7 Lohr, G., Oseguera-Lohr, R., Abbott, T., Capron, W., Howell, C., "Airborne Evaluation and Demonstration of a Time-Based Airborne Inter-Arrival Spacing Tool”, NASA TM-2005-213772

8 Ballin, M., Hoekstra, J., Wing, D., Lohr, G., "NASA Langley and NLR Research of Distributed Air/Ground Traffic Management”, AIAA 2002-5826

9 Callantine, T., Lee, P., Mercer, J., Prevot, T., Palmer, E., "Air and Ground Simulation of Terminal-Area FMS Arrivals With Airborne Spacing and Merging”, $6^{\text {th }}$ ATM Seminar, Baltimore, MD, June 2005

10 Zeitlin, A., Bonnemaison, B., "Managing Criticality of ASAS Applications", $3^{\text {rd }}$ USA/Europe Air Traffic Managmenent R\&D Seminar, 13-16 June 2000, Naples, Italy

11 Mundra, A., Bodoh, C., Domino, D., El-Sahragty, A., Helleberg, J., Smith, A., “Capacity Enhancements in IMC For Airports With Converging Configurations With Knowledge of Aircraft's Expected Final Approach Speeds: A Case Study” MITRE, June 2003, http://www.mitrecaasd.org/library/documents/atm2003_paper70.pdf

12 Grimaund, I., Hoffman, E., Rognin, L., Zeghal, K., "Towards the Use of Spacing Instructions for Sequencing Arrival Flows”, ICAO Operational Datalink Panel, Working Group A Meeting, 29 Oct 2003, Agenda Item 3.

13 Boursier, L., Favennec, B., Hoffman, E., Rognin, L., Vergne, F., Zeghal, K., "Combining Sequencing Tool and Spacing Instructions to Enhance the Management of Arrival Flows of Aircraft”, AIAA 2005-7302

14 Meyn, L., Erzberger, H., “Airport Arrival Capacity Benefits Due to Improved Scheduling Accuracy”, AIAA 2005-7376

${ }^{15}$ Barmore, B., Abbott, T., Capron, W., "Evaluation of Airborne Precision Spacing in a Human-in-the-Loop Experiment", AIAA 2005-7402

16 Barmore, B., Abbott, T., Krishnamurthy, K., “Airborne-Managed Spacing in Multiple Arrival Streams”, ICAS 2004

17 Barmore, B., Abbott, T., Krishnamurthy, K., “An Experimental Validation of Airborne Merging and Spacing Concept", AIAA 2005-7781

18 Weitz, L., Hurtado, J., Barmore, B., Krishnamurthy, K., “An Analysis of Merging and Spacing Operations With Continuous Descent Approaches”, 24 ${ }^{\text {th }}$ Digital Avionics Systems Conference, October 2005

19 Weitz, L., Hurtado, J., Bussink, F., "Increasing Runway Capacity for Continuous Descent Approaches Through Airborne Precision Spacing”, AIAA Guidance, Navigation, and Control Conference, August 2005 